\title{
Tyrosine Kinase Inhibitor Sorafenib Decreases 111In-Girentuximab Uptake in Patients with Clear Cell Renal Cell Carcinoma
}

\author{
Constantijn H.J. Muselaers ${ }^{1,2}$, Alexander B. Stillebroer ${ }^{1}$, Ingrid M.E. Desar ${ }^{3}$, Marye J. Boers-Sonderen ${ }^{3}$, \\ Carla M.L. van Herpen ${ }^{3}$, Mirjam C.A. de Weijert ${ }^{1}$, Johan F. Langenhuijsen ${ }^{1}$, Egbert Oosterwijk ${ }^{1}$, \\ William P.J. Leenders ${ }^{4}$, Otto C. Boerman ${ }^{2}$, Peter F.A. Mulders ${ }^{1}$, and Wim J.G. Oyen ${ }^{2}$ \\ ${ }^{I}$ Radboud university medical center, Department of Urology, Nijmegen, The Netherlands; ${ }^{2}$ Radboud university medical center, \\ Department of Nuclear Medicine, Nijmegen, The Netherlands; ${ }^{3}$ Radboud university medical center, Department of Medical Oncology, \\ Nijmegen, The Netherlands; and ${ }^{4}$ Radboud university medical center, Department of Pathology, Nijmegen, The Netherlands
}

Tyrosine kinase inhibitors (TKIs) have revolutionized the treatment of metastatic clear cell renal cell carcinoma (RCC). Although TKIs have demonstrated good clinical efficacy, the lack of complete responses, the chronic nature of the treatment, and the side effects are clear disadvantages. An interesting new approach in the treatment of clear cell RCC is antibody-mediated therapy with the chimeric anti-carbonic anhydrase IX (CAIX) antibody girentuximab (cG250). As the results of several girentuximab trials become available, the question arises of whether TKI treatment can be combined with girentuximabbased therapy. In this study, we assessed the effect of the widely used TKI sorafenib on the tumor-targeting potential of ${ }^{111} \mathrm{In}$-labeled girentuximab. Methods: ${ }^{111}$ In-girentuximab imaging was performed on 15 patients suspected of having a renal malignancy, with surgery being part of their treatment plan. Of these, 10 patients were treated in a neoadjuvant setting with sorafenib ( $400 \mathrm{mg}$ orally twice daily). Five patients received treatment during $1 \mathrm{wk}$, and 5 patients received treatment during $4 \mathrm{wk}$. In both sorafenib-treated groups, baseline and posttreatment tumor targeting of ${ }^{111}$ In-girentuximab were compared. Surgery was performed $3 \mathrm{~d}$ after the last image acquisition. Five additional patients were included as a control group and had only a single ${ }^{111}$ In-girentuximab injection and scintigraphy without any treatment. Distribution of ${ }^{111} \mathrm{In}$-girentuximab was determined scintigraphically ex vivo in a 1-cm lamella of the resected tumorous kidney. Expression of CAIX and of the vascular marker CD31 was determined immunohistochemically on specimens of both tumor and normal kidney tissue. Results: Treatment with sorafenib resulted in a marked decrease of ${ }^{111} \mathrm{In}$-girentuximab uptake in the tumor in clear cell RCC patients, especially in the group treated for 4 wk (mean change in both sorafenib-treated groups, $-38.4 \%$; range, $+9.1 \%$ to $-79.4 \%$ ). Immunohistochemical analysis showed markedly reduced CD31 expression and vessel density in the sorafenib-treated groups but no differences in CAIX expression between the sorafenib-treated groups and the nontreated patients. Conclusion: Treatment with sorafenib resulted in a treatment duration-dependent significantly decreased uptake of ${ }^{111} \mathrm{In}$-girentumab in clear cell RCC lesions. These results indicate that the efficacy of antibody-mediated treatment or diagnosis modalities is hampered by TKI treatment.

Received Aug. 19, 2013; revision accepted Sep. 26, 2013.

For correspondence or reprints contact: Constantijn H.J. Muselaers, Department of Nuclear Medicine, Radboud University Nijmegen Medical Centre, Geert Grooteplein 8, 6525 GA Nijmegen, The Netherlands.

E-mail: s.muselaers@uro.umcn.nl

Published online Jan. 6, 2014.

COPYRIGHT $\odot 2014$ by the Society of Nuclear Medicine and Molecular Imaging, Inc.
Key Words: CAIX; girentuximab; imaging; renal cell carcinoma; tyrosine kinase inhibitor

J Nucl Med 2014; 55:242-247

DOI: 10.2967/jnumed.113.131110

$\mathbf{T}$ yrosine kinase inhibitors (TKIs) have revolutionized the treatment of renal cell carcinoma (RCC) during the last $5 \mathrm{y}$. Sunitinib, sorafenib, and pazopanib are now standard care in patients with advanced disease (1-3). Although excellent results for progression-free survival have been achieved, there are limitations to the use of TKIs. First, these agents have a cytostatic rather than cytotoxic effect, and thus complete responses are rarely seen. Second, severe side effects such as hypertension, nausea, handfoot skin reactions, and diarrhea can occur, and sustained lowgrade toxicity can lead to dose adjustments. Third, the long-term effects of these agents are largely unknown (4). Moreover, TKI treatment is chronic, and cessation of treatment may lead to flare-up of the disease, possibly due to rapid neovascularization (5). A new alternative approach in the treatment of clear cell RCC is antibody-mediated therapy using the chimeric anti-carbonic anhydrase IX (CAIX) antibody girentuximab (cG250). CAIX is an antigen ubiquitously expressed in clear cell RCC $(6,7)$, but expression in normal tissues is low or absent (8). The high and specific expression in clear cell RCC makes CAIX an excellent target for antibody-mediated therapy. Girentuximab has been studied in radioimmunotherapy trials in patients with metastatic disease (9-15) and is currently studied in trials in an adjuvant setting (ARISER, or Adjuvant RENCAREX [Wilex AG] Immunotherapy Trial to Study Efficacy in Nonmetastasised Renal Cell Carcinoma) (16). Because we recently published promising results for the most recent therapy trial (15), the question arises of whether antibody-mediated treatment can be combined with TKI treatment.

In this trial, we aimed to determine the effect of the widely used TKI sorafenib on tumor targeting of ${ }^{111}$ In-labeled chimeric antiCAIX monoclonal antibody girentuximab in preoperative patients presenting with a renal mass suspected of being RCC. To assess the effect of the duration of the treatment, patients were treated either during $1 \mathrm{wk}$ or during $4 \mathrm{wk}$. 


\section{MATERIALS AND METHODS}

\section{Study Design}

Fifteen patients with renal masses suspected of being RCC and for whom surgery was planned were included in this study.

Before inclusion at baseline (day -14 to day 0 ), a physical examination (including vital signs and weight) was performed, biochemical laboratory parameters were assessed, and a resting electrocardiogram was made. The study was approved by the institutional review board (CMO Arnhem-Nijmegen). Written informed consent was obtained from every patient. This trial is registered on www.clinicaltrials.gov, number NCT00602862.

Two groups of 5 patients were infused with ${ }^{111}$ In-labeled girentuximab (100 MBq; $5 \mathrm{mg}$ ), and whole-body and SPECT images were obtained $7 \mathrm{~d}$ later. Imaging was followed by treatment with sorafenib (Nexavar [Bayer], $400 \mathrm{mg}$ twice a day orally) for $1 \mathrm{wk}$ or $4 \mathrm{wk}$ (Fig. 1). All sorafenibtreated patients underwent a second injection with ${ }^{111}$ In-girentuximab and imaging $7 \mathrm{~d}$ after injection. The sorafenib treatment was discontinued on the day of the last scan in both groups. Five patients served as a control group and received a single infusion of ${ }^{111}$ In-girentuximab $10 \mathrm{~d}$ before nephrectomy and were scanned $7 \mathrm{~d}$ later but did not receive sorafenib treatment. The study design is summarized in Figure 1.

\section{Study Drugs}

Sorafenib was administered orally in a dosage of $400 \mathrm{mg}$ twice a day. Dose interruptions and reductions were allowed when there were adverse events of grade III or higher according to the Common Terminology Criteria for Adverse Events, version 3.0 (National Cancer Institute).

For scintigraphic imaging, diethylenetriaminepentaacetic acid (DTPA) was conjugated to girentuximab (cG250; Wilex AG) at pH 9.5. A 50-fold molar excess of isothiocyanato-benzyl-DTPA was used to obtain a molar substitution ratio of 0.5-2.0. After conjugation, the unconjugated DTPA was removed by extensive dialysis against a $0.25 \mathrm{M}$ ammonium acetate buffer, $\mathrm{pH}$ 5.4. Aliquots of the girentuximab-DTPA solution were placed into sterile vials $(5 \mathrm{mg}$ in $1.0 \mathrm{~mL})$ and stored at $-20^{\circ} \mathrm{C}$ until use. This procedure has been previously described in more detail (14). After inclusion of a patient, the girentuximab-DTPA conjugate was labeled with $100 \mathrm{MBq}$ of ${ }^{111}$ In (Covidien). The volume of ${ }^{111}$ In-DTPAgirentuximab was adjusted to $10 \mathrm{~mL}$ with $\mathrm{NaCl}, 0.9 \%$.
Radiochemical purity was determined by instant thin-layer chromatography. All preparations used in this study exceeded $95 \%$.

\section{Imaging and Quantitative Image Analysis}

On the basis of previous studies, whole-body scans were acquired $7 \mathrm{~d}$ after injection using a double-head $\gamma$ camera (E.cam; Siemens Inc.) equipped with parallel-hole medium-energy collimators (symmetric $15 \%$ window over 172 and $247 \mathrm{keV}$ ) and a scan speed of $4 \mathrm{~cm} / \mathrm{min}$ (17). The sorafenib-treated groups were injected and scanned twice; the same imaging procedures were used for both scans.

In the group that was treated with sorafenib for $1 \mathrm{wk},{ }^{111}$ In-girentuximab was administered at $11 \mathrm{~d}$ before the start of sorafenib treatment and on the day that treatment began. Treatment with sorafenib was started $4 \mathrm{~d}$ after the first ${ }^{111}$ In-girentuximab image was acquired. Each time, scintigraphic imaging was performed $7 \mathrm{~d}$ after injection of ${ }^{111} \mathrm{In}$-girentuximab (Fig. 1).

In the group that received $4 \mathrm{wk}$ of sorafenib, ${ }^{111}$ In-girentuximab was administered at $7 \mathrm{~d}$ before the start of sorafenib treatment and after $21 \mathrm{~d}$ of treatment, each time followed by scintigraphy $7 \mathrm{~d}$ after injection. The sorafenib treatment was stopped in both groups on the day of the last scan, and $3 \mathrm{~d}$ later a (partial) tumor nephrectomy was performed (Fig. 1).

In the control group, patients were intravenously infused with ${ }^{111} \mathrm{In}$ girentuximab $10 \mathrm{~d}$ before nephrectomy and scanned $7 \mathrm{~d}$ after injection of the radiolabeled antibody.

Whole-body planar images were analyzed quantitatively, as described by Visser et al. (18). Regions of interest were drawn around tumors and normal kidney. Targeting was expressed as the percentage injected dose per tissue weight $(\% \mathrm{ID} / \mathrm{g})$, assuming a tissue density of $1.0 \mathrm{~g} / \mathrm{mL}$. Tumor volumes were determined using Inveon Research Workplace software (Siemens Inc.).

\section{Processing of the Surgical Specimen}

After nephrectomy, a 1-cm-thick slice of the resected tissue containing both tumor and normal tissue was scanned on the $\gamma$ camera, using only a single detector. After imaging, the slice was cut into $1-\mathrm{cm}^{3}$ blocks. Tissue blocks were weighed, and radioactivity in each sample was determined in a well-type $\gamma$ counter (1480 Wizard; LKB/ Wallace, Perkin-Elmer). After quantitative analysis, the tissue blocks were processed for immunohistochemical analysis.

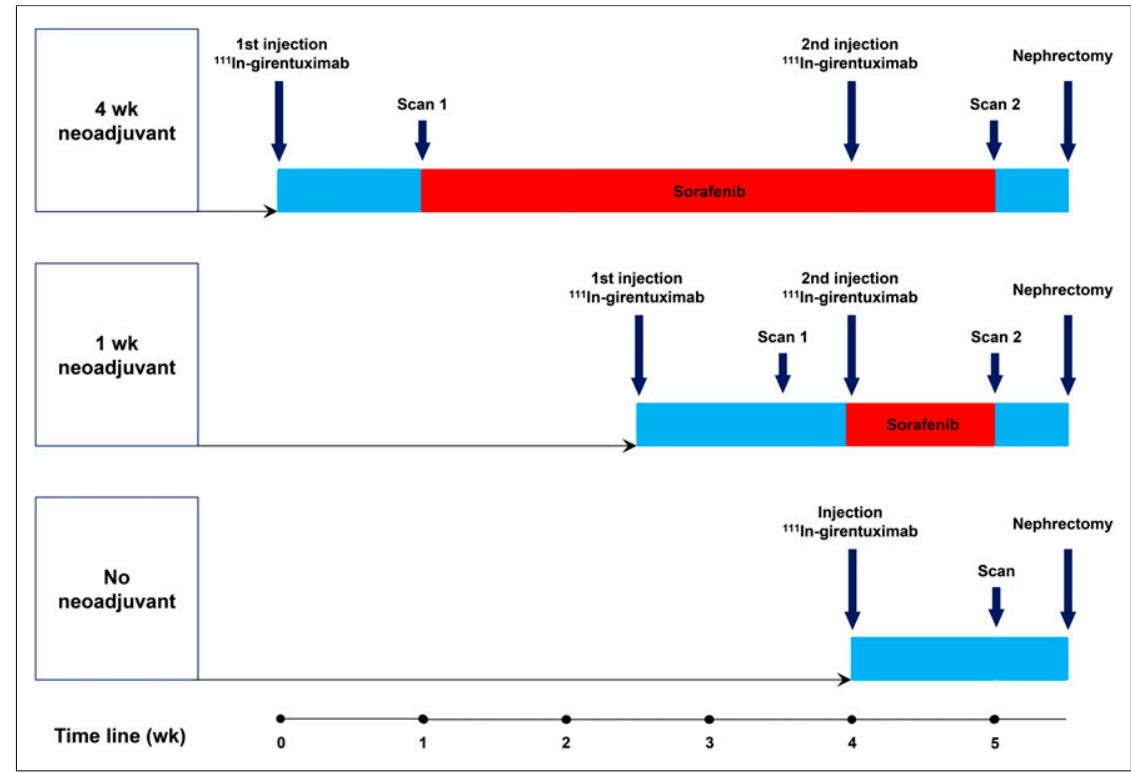

FIGURE 1. Study design.

\section{Immunohistochemical Analysis}

CAIX expression was detected by staining with anti-CAIX mouse monoclonal antibody M75, a hybridoma supernatant obtained from the HB-11128 ATCC cell line. CD31 staining was performed with murine anti-CD31 monoclonal antibody JC70A (Dako). After staining, expression of CAIX and CD31 was scored by 4 independent observers on a scale ranging from undetectable $(-)$ to low $( \pm)$, moderate $(+)$, high $(++)$, and very high $(+++)$.

\section{Statistics}

A nonparametric Wilcoxon signed-ranked test was performed to assess the change in ${ }^{111}$ In-girentuximab uptake in the tumor before and after sorafenib treatment. A $P$ value of less than 0.05 was considered significant.

\section{RESULTS}

\section{Patients}

Patient characteristics are summarized in Table 1. Ten patients were treated with 


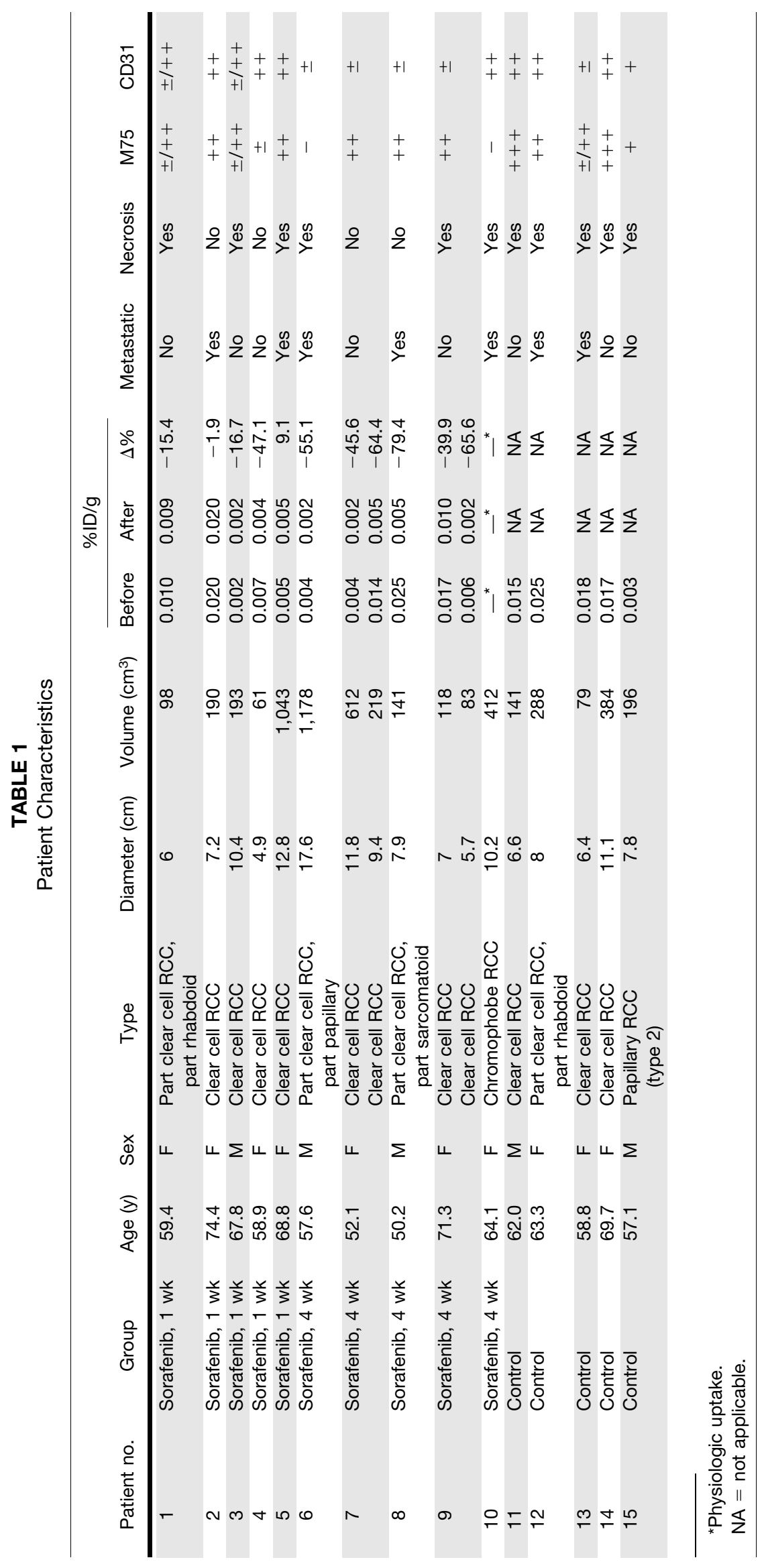


sorafenib ( 7 women and 3 men; median age, 62.4 y; range, 50.2$74.4 \mathrm{y}$ ). The tumors of 9 of these patients were clear cell RCC as determined by pathology (patients 2, 3, 4, 5, 7, and 9) or contained a clear cell RCC component (patients 1, 6, and 8). Patient 1 had a poorly differentiated tumor, partly clear cell RCC and partly rhabdoid RCC; patient 6 a partly clear cell RCC and partly papillary dedifferentiated tumor; and patient 8 a partly clear cell RCC with sarcomatoid dedifferentiated tumor. Patient 10 did not have clear cell RCC but a tumor of the chromophobe subtype.

In the control group ( 3 women and 2 men; median age, $62.2 \mathrm{y}$; range, 57.1-69.7 y), histopathologic examination revealed RCC in all patients. Of these patients, patient 11,13 , and 14 had the clear cell RCC subtype. The surgical specimen of patient 12 showed clear cell RCC, but a part was rhabdoid-dedifferentiated. The specimen of patient 15 revealed a type 2 papillary RCC (Table 1 ).

Treatment with sorafenib was generally well tolerated. Reported side effects were grade 3 skin toxicity according to the Common Terminology Criteria for Adverse Events (version 3; National Cancer Institute) in 2 patients, grade 2 diarrhea in 1 patient, and grade 2 stomatitis in 2 patients. No dose reductions or interruptions were necessary.

\section{Scintigraphy}

In all patients with later-proven clear cell RCC, the renal tumors before neoadjuvant treatment were readily visualized with ${ }^{111}$ Ingirentuximab. The results of the quantitative analysis are summarized in Table 1. Imaging of the 1-cm-thick slices of the resected tumorous tissue, followed by histologic analysis, revealed that ${ }^{111} \mathrm{In}$ girentuximab accumulated in areas of vital tumor tissue whereas normal kidney and necrotic or non-clear cell RCC parts showed much lower uptake. In one patient, the lung metastases as observed on CT were also visualized with scintigraphy (patient 8, Fig. 2). In this patient, the uptake in the largest metastasis decreased by $74.8 \%$ after 4 wk of sorafenib treatment. Patients 7 and 9 were diagnosed with bilateral renal masses and had girentuximab uptake in both lesions before treatment (patient 9, shown in Fig. 3). Histologic analysis of the surgical specimens revealed that all these lesions were of the clear cell type (Table 1).

The mean ${ }^{111} \mathrm{In}$-girentuximab uptake in clear cell RCC renal tumors before sorafenib treatment was $0.013 \% \mathrm{ID} / \mathrm{g}$ (range, 0.002-0.025 $\% \mathrm{ID} / \mathrm{g}$ ). After $1 \mathrm{wk}$ of sorafenib treatment, the mean uptake decreased to $0.008 \% \mathrm{ID} / \mathrm{g}$ (range, $0.002-0.02 \% \mathrm{ID} / \mathrm{g}$ ), indicating a mean change of $-14.4 \%$ (range, $+9.1 \%$ to $-47.1 \%, P=0.225$ ). After 4 wk of sorafenib treatment, the mean uptake decreased to $0.004 \% \mathrm{ID} / \mathrm{g}$ (range, $0.002-0.01 \% \mathrm{ID} / \mathrm{g}$ ), which is equivalent to a mean change of $-58.3 \%$ (range, $-39.9 \%$ to $-79.4 \%, P=0.028$ ) based on 6 lesions in 4 patients.

The tumors of all 5 patients who served as controls showed accumulation of ${ }^{111}$ In-girentuximab, with a mean uptake of 0.018 $\% \mathrm{ID} / \mathrm{g}$. Uptake of ${ }^{111} \mathrm{In}$-girentuximab was also found in the papillary RCC in patient 15 , albeit relatively low compared with the mean baseline uptake in the clear cell RCC tumors $(0.003 \% \mathrm{ID} / \mathrm{g}$ vs. a mean of $0.018 \% \mathrm{ID} / \mathrm{g}$ ).

\section{Immunohistochemical Analysis}

The results of the immunohistochemical analyses of the viable parts of the tumors are presented in Table 1. All clear cell RCC tumor specimens expressed CAIX (Table 1). Very low CAIX expression was also found in a vital tumor region of the surgical specimen of the papillary subtype from patient 15 (not shown).

Representative examples of postnephrectomy tumor specimens of each of the 3 groups are shown in Figure 4. From each tumor,

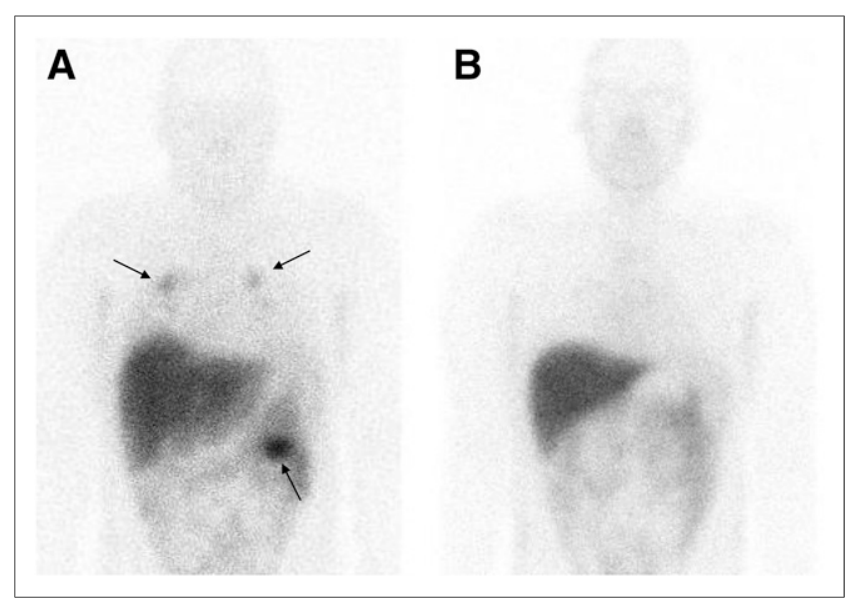

FIGURE 2. Anterior planar images of patient 8 with renal mass and lung metastases at baseline $(A)$ and after $4 \mathrm{wk}$ of sorafenib treatment $(B)$. Clear targeting is seen of ${ }^{111}$ In-girentuximab in renal mass on left side and lung metastases at baseline (arrows). Decreased targeting is observed after $4 \mathrm{wk}$ of sorafenib treatment.

multiple tumor blocks with the highest ${ }^{111}$ In uptake per gram of tissue (as determined in a $\gamma$ counter) were used. High expression of CD31 was found in all tumors in the control group. As expected, sorafenib treatment induced a marked reduction of vessel density, as observed by CD31 immunohistochemistry. This effect was more pronounced in the group that was treated for $4 \mathrm{wk}$ than in the group that was treated for $1 \mathrm{wk}$, suggesting that the decrease in vessel density was related to the duration of treatment.

\section{DISCUSSION}

This study confirmed previous studies showing that clear cell RCC lesions can be visualized with ${ }^{111}$ In-girentuximab imaging $(17,19)$. High and specific uptake was seen in tumor lesions in all patients with clear cell RCC. Most importantly, we found that accumulation of girentuximab in clear cell RCC lesions was markedly reduced after sorafenib treatment in most patients.

The effect of sorafenib on girentuximab uptake was more pronounced in patients who were treated with sorafenib for $4 \mathrm{wk}$ than in the group treated for $1 \mathrm{wk}(-58.3 \%$ vs. $-14.4 \%)$. All clear cell

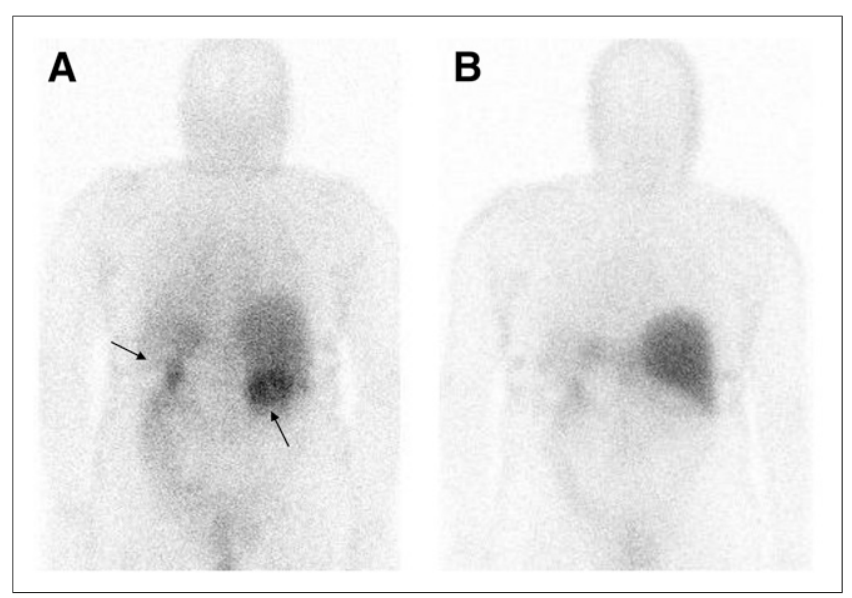

FIGURE 3. Posterior planar images of patient 9 with bilateral renal mass at baseline (A) and after $4 \mathrm{wk}$ of sorafenib treatment (B). Clear targeting of ${ }^{111}$ In-girentuximab is seen in both lesions at baseline (arrows). Decreased targeting is observed after $4 \mathrm{wk}$ of sorafenib treatment. 


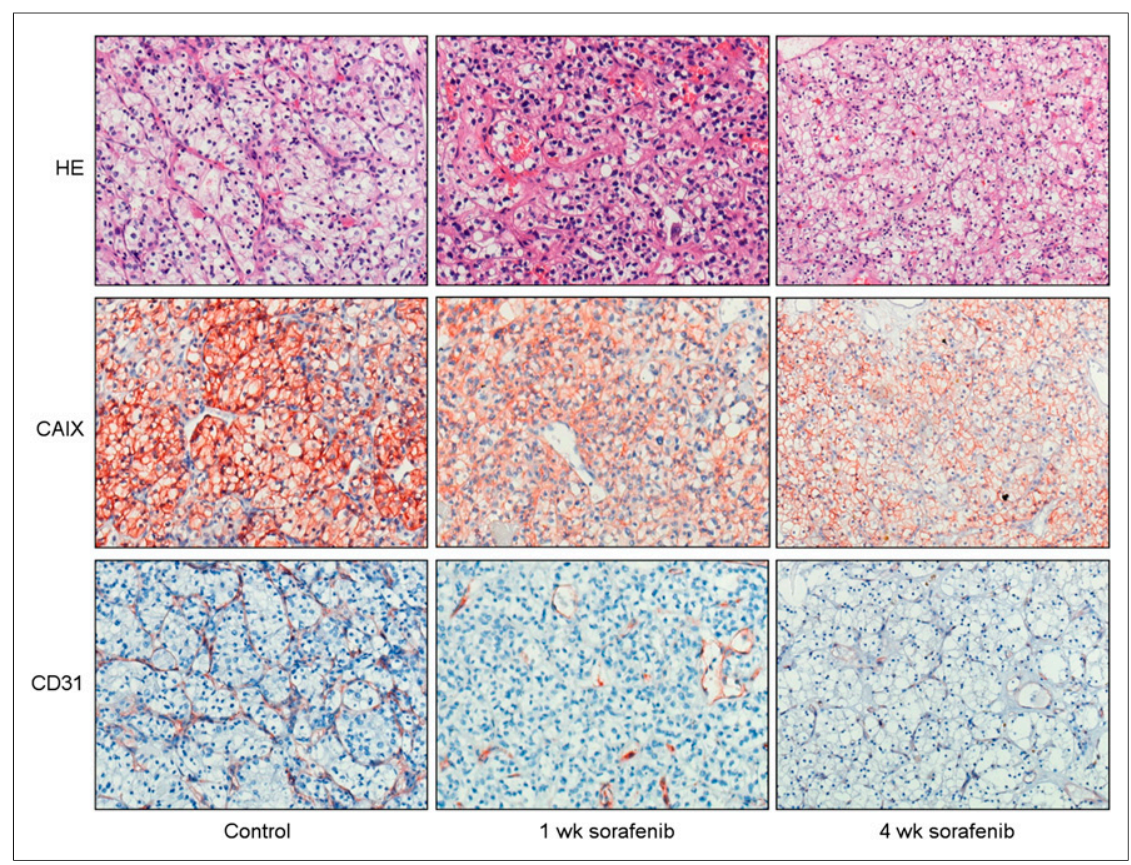

FIGURE 4. Immunohistochemical analyses of vital tumor specimens from different groups (magnification, $\times 20$ ). Neoadjuvant treatment with sorafenib resulted in enhanced necrosis (hematoxylin and eosin) and decreased vessel density (CD31) but had no effect on CAIX expression.

antibodies (immuno-PET or immuno-SPECT) may similarly be affected by TKI treatment. Currently, a clinical trial is ongoing that focuses on the potential of ${ }^{124}$ I-girentuximab immuno-PET to detect early treatment response to sunitinib (26). It will be interesting to see whether this study also indicates that TKIs interfere with the uptake of girentuximab and whether it is possible to evaluate the response to TKI treatment with girentuximab-based imaging.

There are some limitations to our study. First, there was a 3-d interval between the discontinuation of the sorafenib treatment and the (partial) nephrectomy. Therefore, the immunohistochemical findings may not fully reflect the actual effect caused by sorafenib, though significant histologic changes in this short interval are unlikely.

Second, no biopsies of the renal lesions were taken at baseline, and therefore no intrapatient comparison of pre- and posttreatment tissue samples could be performed. In addition, vascular density may vary largely within and between RCC tumors. However, the CD31 expression in the samples from all patients treated with sorafenib for $4 \mathrm{wk}$

RCC tumor specimens in the current study were positive for CAIX, and we found no decrease in CAIX expression after the TKI treatment, indicating that sorafenib treatment did not down-regulate CAIX expression. We did observe decreased vessel densities in the sorafenib-treated groups when compared with the untreated group, making it highly likely that the reduced vessel density in the treated tumors results in poor perfusion and decreased uptake of ${ }^{111} \mathrm{In}$-girentuximab. The decrease in vessel density was most prominent after $4 \mathrm{wk}$ of treatment (Table 1; Fig. 4). These results are in concordance with previous work of our group showing a similar effect of sorafenib on bevacizumab uptake (20) and with preclinical studies with other antibodies $(21,22)$. Alternatively, the reduced ${ }^{111}$ In-girentuximab uptake could also be due to vascular normalization after sorafenib treatment (23). Vascular normalization may have led to decreased vessel permeability, which could also have led to reduced accumulation of the antibody in the tumor. We also found CAIX expression in a patient presenting with a papillary RCC subtype (patient 15). Although CAIX expression has been reported in papillary RCC subtypes, the expression is much lower than in clear cell RCC, in terms of both incidence and level of expression $(8,24)$. Strikingly, the CAIX expression in this particular patient was found in a vital tumor region within a necrotic part of the surgical specimen (Supplemental Fig. 1, available at http://jnm.snmjournals.org), suggesting that this particular expression pattern is hypoxia-driven (25).

The TKI-induced decrease of antibody uptake in the clear cell RCC lesions has major implications for future therapeutic regimens combining both TKIs and antibody-based treatments (either cold or radiolabeled). The results of the present study indicate that antibody-mediated treatment should preferably be given before TKI treatment. Alternatively, the antibody could be administered after TKI treatment has been stopped. The interval required between TKI cessation and antibody treatment still needs to be determined in future studies. In addition, the diagnostic performance of radiolabeled was lower than in the other samples, suggesting that the lower vessel density is a direct effect of the treatment. Although the changes in CD31 expression after sorafenib treatment were evident (Fig. 4), microvessel densities in the treated groups could not be reliably quantified. In desmoplastic areas of treated tumors, we often observed cytoplasmic CD31 expression in nonendothelial cells. This phenomenon has been documented before and has been linked to the phenomenon of vasculogenic mimicry $(27,28)$. Third, the current trial was not designed to assess the duration of the sorafenibinduced decreased antibody uptake. It remains to be investigated for how long antibody targeting to the tumor remains at lower levels after cessation of sorafenib treatment. Data on this subject are limited. There are animal data suggesting increased antibody uptake after discontinuation of sunitinib treatment $(21,22)$, presumably due to rapid rebound vascularization. To date, no human data are available yet. Enhanced uptake of girentuximab shortly after discontinuation of TKI treatment is an interesting feature for further investigation because it could be an important step in the development of successful radioimmunotherapy strategies for clear cell RCC.

\section{CONCLUSION}

This study confirmed that clear cell RCC lesions can be identified with ${ }^{111}$ In-girentuximab scintigraphy. Moreover, we demonstrated that treatment with sorafenib markedly reduces the targeting of ${ }^{111}$ In-girentuximab in clear cell RCC lesions, suggesting that the effect of antibody-mediated treatment modalities would be profoundly hampered when directly combined with TKI treatment. Further research to evaluate the duration of this effect after discontinuation of TKI treatment is needed.

\section{DISCLOSURE}

The costs of publication of this article were defrayed in part by the payment of page charges. Therefore, and solely to indicate this 
fact, this article is hereby marked "advertisement" in accordance with 18 USC section 1734. Peter F.A. Mulders, Wim J.G. Oyen, Otto C. Boerman, and Egbert Oosterwijk serve or have served on an advisory board for Wilex AG, Munich, Germany. Sorafenib was provided by Bayer. Girentuximab was provided by Wilex. No other potential conflict of interest relevant to this article was reported.

\section{ACKNOWLEDGMENTS}

We thank Maichel van Riel and Miranda van de Veerdonk for their help with the labeling of the antibody.

\section{REFERENCES}

1. Motzer RJ, Hutson TE, Tomczak P, et al. Sunitinib versus interferon alfa in metastatic renal-cell carcinoma. N Engl J Med. 2007;356:115-124.

2. Escudier B, Eisen T, Stadler WM, et al. Sorafenib in advanced clear-cell renalcell carcinoma. N Engl J Med. 2007;356:125-134.

3. Sternberg CN, Davis ID, Mardiak J, et al. Pazopanib in locally advanced or metastatic renal cell carcinoma: results of a randomized phase III trial. J Clin Oncol. 2010;28:1061-1068.

4. Hartmann JT, Haap M, Kopp HG, Lipp HP. Tyrosine kinase inhibitors: a review on pharmacology, metabolism and side effects. Curr Drug Metab. 2009;10: $470-481$.

5. Desar IM, Mulder SF, Stillebroer AB, et al. The reverse side of the victory: flare up of symptoms after discontinuation of sunitinib or sorafenib in renal cell cancer patients-a report of three cases. Acta Oncol. 2009;48:927-931.

6. Oosterwijk E, Ruiter DJ, Hoedemaeker PJ, et al. Monoclonal antibody G 250 recognizes a determinant present in renal-cell carcinoma and absent from normal kidney. Int J Cancer. 1986;38:489-494.

7. Bui MH, Seligson D, Han KR, et al. Carbonic anhydrase IX is an independent predictor of survival in advanced renal clear cell carcinoma: implications for prognosis and therapy. Clin Cancer Res. 2003;9:802-811.

8. Leibovich BC, Sheinin Y, Lohse CM, et al. Carbonic anhydrase IX is not an independent predictor of outcome for patients with clear cell renal cell carcinoma. J Clin Oncol. 2007;25:4757-4764.

9. Divgi CR, Bander NH, Scott AM, et al. Phase I/II radioimmunotherapy trial with iodine-131-labeled monoclonal antibody G250 in metastatic renal cell carcinoma. Clin Cancer Res. 1998;4:2729-2739.

10. Steffens MG, Boerman OC, de Mulder PH, et al. Phase I radioimmunotherapy of metastatic renal cell carcinoma with ${ }^{131} \mathrm{I}$-labeled chimeric monoclonal antibody G250. Clin Cancer Res. 1999;5(suppl)3268s-3274s.

11. Divgi CR, O'Donoghue JA, Welt S, et al. Phase I clinical trial with fractionated radioimmunotherapy using ${ }^{131} \mathrm{I}$-labeled chimeric G250 in metastatic renal cancer. J Nucl Med. 2004;45:1412-1421.

12. Brouwers AH, Buijs WC, Mulders PF, et al. Radioimmunotherapy with $\left[{ }^{131} \mathrm{I}\right]$ cG250 in patients with metastasized renal cell cancer: dosimetric analysis and immunologic response. Clin Cancer Res. 2005;11(suppl):7178s-7186s.
13. Brouwers AH, Mulders PF, de Mulder PH, et al. Lack of efficacy of two consecutive treatments of radioimmunotherapy with ${ }^{131} \mathrm{I}-\mathrm{cG} 250$ in patients with metastasized clear cell renal cell carcinoma. J Clin Oncol. 2005;23:6540-6548.

14. Brouwers AH, van Eerd JE, Frielink C, et al. Optimization of radioimmunotherapy of renal cell carcinoma: labeling of monoclonal antibody cG250 with ${ }^{131} \mathrm{I}$, ${ }^{90} \mathrm{Y},{ }^{177} \mathrm{Lu}$, or ${ }^{186} \mathrm{Re}$. J Nucl Med. 2004;45:327-337.

15. Stillebroer AB, Boerman OC, Desar IM, et al. Phase 1 radioimmunotherapy study with lutetium 177-labeled anti-carbonic anhydrase IX monoclonal antibody girentuximab in patients with advanced renal cell carcinoma. Eur Urol. 2013;64:478-485.

16. A randomized, double blind phase III study to evaluate adjuvant cG250 treatment versus placebo in patients with clear cell RCC and high risk of recurrence (ARISER). ClinicalTrials.gov Web site. http://www.clinicaltrials.gov/ct2/show/ NCT00087022. Published July 8, 2004. Updated May 21, 2013. Accessed December 11, 2013.

17. Brouwers AH, Buijs WC, Oosterwijk E, et al. Targeting of metastatic renal cell carcinoma with the chimeric monoclonal antibody G250 labeled with ${ }^{131} \mathrm{I}$ or ${ }^{111}$ In: an intrapatient comparison. Clin Cancer Res. 2003;9(suppl):3953S-3960S.

18. Visser E, Postema E, Boerman O, Visschers J, Oyen W, Corstens F. Software package for integrated data processing for internal dose assessment in nuclear medicine (SPRIND). Eur J Nucl Med Mol Imaging. 2007;34:413-421.

19. Muselaers $\mathrm{CH}$, Boerman OC, Oosterwijk E, Langenhuijsen JF, Oyen WJ, Mulders PF. Indium-111-labeled girentuximab immunoSPECT as a diagnostic tool in clear cell renal cell carcinoma. Eur Urol. 2013;63:1101-1106.

20. Desar IM, Stillebroer AB, Oosterwijk E, et al. ${ }^{111}$ In-bevacizumab imaging of renal cell cancer and evaluation of neoadjuvant treatment with the vascular endothelial growth factor receptor inhibitor sorafenib. J Nucl Med. 2010;51:1707-1715.

21. Nagengast WB, Lub-de Hooge MN, Oosting SF, et al. VEGF-PET imaging is a noninvasive biomarker showing differential changes in the tumor during sunitinib treatment. Cancer Res. 2011;71:143-153.

22. Oosterwijk-Wakka JC, Kats-Ugurlu G, Leenders WP, et al. Effect of tyrosine kinase inhibitor treatment of renal cell carcinoma on the accumulation of carbonic anhydrase IX-specific chimeric monoclonal antibody cG250. BJU Int. 2011;107:118-125.

23. Jain RK. Normalization of tumor vasculature: an emerging concept in antiangiogenic therapy. Science. 2005;307:58-62.

24. Ross H, Martignoni G, Argani P. Renal cell carcinoma with clear cell and papillary features. Arch Pathol Lab Med. 2012;136:391-399.

25. Russell J, Carlin S, Burke SA, Wen B, Yang KM, Ling CC. Immunohistochemical detection of changes in tumor hypoxia. Int J Radiat Oncol Biol Phys. 2009;73: 1177-1186.

26. Evaluate the utility of ${ }^{124} \mathrm{I}-\mathrm{cG} 250$ for the early detection of response to sunitinib in patients with metastatic renal cell carcinoma. ClinicalTrials.gov Web site. http://clinicaltrials.gov/ct2/show/NCT01582204. Published April 18, 2012. Updated June 25, 2013. Accessed December 11, 2013.

27. Francescone R, Scully S, Bentley B, et al. Glioblastoma-derived tumor cells induce vasculogenic mimicry through Flk-1 protein activation. $J$ Biol Chem. 2012;287:24821-24831.

28. Scully S, Francescone R, Faibish M, et al. Transdifferentiation of glioblastoma stem-like cells into mural cells drives vasculogenic mimicry in glioblastomas. J Neurosci. 2012;32:12950-12960. 